\title{
Carnets
}

Revue électronique d'études françaises de l'APEF

Deuxième série - 9 | 2017

Reconnaissances et légitimité en français

\section{Complexités des prix littéraires et littérature française ou en français}

Paradoxes, mythes et enjeux d'une certaine idée de la littérature

Marie-Manuelle da Silva et Eduarda Keating

\section{CpenEdition}

Journals

Édition électronique

URL : http://journals.openedition.org/carnets/2042

DOI : $10.4000 /$ carnets. 2042

ISSN : 1646-7698

Éditeur

APEF

Référence électronique

Marie-Manuelle da Silva et Eduarda Keating, «Complexités des prix littéraires et littérature française ou en français », Carnets [En ligne], Deuxième série - 9 | 2017, mis en ligne le 31 janvier 2017, consulté le 05 mai 2019. URL : http://journals.openedition.org/carnets/2042 ; DOI : 10.4000/carnets.2042

Ce document a été généré automatiquement le 5 mai 2019.

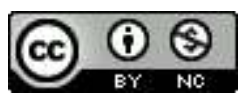

Carnets est mis à disposition selon les termes de la licence Creative Commons - Atribution - Pas d'utilisation commerciale 4.0 International. 


\title{
Complexités des prix littéraires et littérature française ou en français
}

\author{
Paradoxes, mythes et enjeux d'une certaine idée de la littérature
}

Marie-Manuelle da Silva et Eduarda Keating

1 L'attribution du Prix Nobel de Littérature à Patrick Modiano en 2014 après l'attribution du même prix à JM Le Clézio en 2008, a suscité toutes sortes de commentaires de la part des promoteurs de la culture et de la littérature françaises dans le monde. Ces deux prix décernés à des auteurs français dans un laps de temps relativement réduit, à l'échelle du Nobel en tout cas, ont parfois été interprétés comme le retour de la reconnaissance internationale de la fiction en langue française et de la prégnance de la langue française dans le monde. Ceci établissant une sorte d'amalgame ambigüe entre «France- français » et « langue française » ou « fiction en français ».

2 C'est cette tension, qui traverse le champ littéraire français, dans lequel les prix littéraires assument traditionnellement une importance considérable que nous souhaiterions tenter d'examiner ici.

3 En 2007 déjà, un an avant l'attribution du Prix Nobel à Jean-Marie Le Clézio, la publication du Manifeste pour une littérature-monde en français développait des considérations à ce sujet, ambigües et controversées elles aussi. On s'en souvient car ce Manifeste a été abondamment commenté depuis, c'est précisément les Prix littéraires qui en étaient à l'origine :

Plus tard, on dira peut-être que ce fut un moment historique : le Goncourt, le Grand Prix du roman de l'Académie française, le Renaudot, le Femina, le Goncourt des lycéens, décernés le même automne à des écrivains d'outre-France. Simple hasard d'une rentrée éditoriale concentrant par exception les talents venus de la "périphérie", simple détour vagabond avant que le fleuve revienne dans son lit? Nous pensons, au contraire : révolution copernicienne. Copernicienne, parce qu'elle révèle ce que le milieu littéraire savait déjà sans l'admettre: le centre, ce point depuis lequel était supposée rayonner une littérature franco-française, n'est plus le centre (...) le centre, nous disent les prix d'automne, est désormais partout, aux quatre coins du monde. Fin de la francophonie. Et naissance d'une littératuremonde en français. (Pour une « littérature-monde » en français, 2007) 
Et un peu plus loin :

Le monde revient. Et c'est la meilleure des nouvelles. N'aura-t-il pas été longtemps le grand absent de la littérature française ? Le monde, le sujet, le sens, l'histoire, le « référent » : pendant des décennies, ils auront été mis « entre parenthèses » par les maîtres-penseurs, inventeurs d'une littérature sans autre objet qu'elle-même, faisant, comme il se disait alors, «sa propre critique dans le mouvement même de son énonciation » (...) Plutôt que de se frotter au monde pour en capter le souffle, les énergies vitales, le roman, en somme, n'avait plus qu'à se regarder écrire. ( ibidem)

Ce Manifeste peut être lu comme prise de position contre l'impérialisme culturel français, qu'il conforte, paradoxalement, lorsqu'il présente la razzia des Prix d'Automne attribués à des auteurs "francophones " comme un événement fondamental pour le champ littéraire français et qu'il renforce indirectement lorsqu'il reconnaît les prix parisiens comme des instances de légitimation matricielle de ce qui constituerait « une littératuremonde en français ».

6 Ce texte ne fait que reprendre les jalons du " grand récit » de la littérature française dans un champ littéraire que nous avons choisi d'observer sous le prisme des prix littéraires et des auteurs ayant remporté le Nobel de littérature. L'hypothèse que nous souhaiterions avancer ici est que les prix français consacrent des auteurs que le Nobel semble légitimer a posteriori.

7 En reprenant la périodisation du champ littéraire proposée par Alain Viala (Viala, 1985), on peut décrire l'organisation du "grand récit» de la littérature française autour de l'apogée, puis du déclin d'une certaine conception de la littérature. En effet, on a d'abord assisté à l'autonomisation progressive des catégories avec lesquelles les écrivains ont pensé leurs propres pratiques d'écriture avec, dès le XvII ${ }^{\mathrm{e}}$ siècle, l'émergence d'une valeur proprement littéraire, exempte de toute considération religieuse, politique ou économique. Cette autonomie a culminé durant la seconde moitié du xIx ${ }^{e}$ siècle; la professionnalisation de l'écrivain rendant possible «l'art pour l'art» et le «livre sur rien » cher à Flaubert. Enfin, une désagrégation a eu lieu dans la seconde moitié du $\mathrm{xx}^{\mathrm{e}}$, avec la redistribution du champ français, la rentabilisation du capital symbolique de l'écrivain et la médiatisation de la littérature et de la critique.

8 L'histoire des prix en France a suivi le développement de la "nation littéraire » où ils prolifèrent depuis plus d'un siècle et font partie de ce que Sylvie Ducas (2006) appelle une "économie du prestige " adossée aux "mythologie romantiques du Grand Écrivain » (Ducas, 2010) et aux modalités de production de la valeur littéraire.

9 La création des prix s'inscrit dans le temps long de l'histoire des luttes pour le monopole de la définition légitime de l'écrivain qui ont marqué le $\mathrm{xx}^{\mathrm{e}}$ siècle, jusqu'alors aux mains de l'Académie Française - l'institution s'assumant comme dépositaire de la langue nationale dont elle a garanti l'exemplarité durant des siècles, défendant le grand style et les grands genres (Ducas 2010).

Le prix Goncourt naît en 1886 (le premier prix est décerné en 1903). Le plus ancien des prix français, "héritier direct des cénacles et des banquets lettrés du XVIII ${ }^{\mathrm{e}}$ siècle " (Ducas 2010) est institué par Edmond de Goncourt qui a légué sa fortune pour la création d'une académie qui attribue une rente à vie à ces académiciens pour les protéger de l'industrie des lettres et leur épargner les besognes alimentaires ou les promiscuités avec la presse. Les auteurs, autrement dit le jury, étaient choisis parmi les avant-gardes de production restreinte, en prose. L'affrontement emblématique des Dix académiciens de 
l'Académie Goncourt et des Immortels de l'Académie Française engageait une redéfinition des hiérarchies esthétiques entre vers et prose à une époque où il était devenu possible à la prose romanesque d'imposer son hégémonie.

11 Au-delà de la question de l'indépendance du jury à l'origine de nombreuses polémiques, l'ironie du Goncourt est de servir les intérêts d'une industrie des lettres et du livre qu'il entendait à l'inverse combattre. Les prémices d'une crise du modèle académique sont décelables dans la prolifération immédiate de prix littéraires concurrents, à commencer par le Prix du Roman de l'Académie Française :

(...) l'Académie française, s'étant décidée sur le tard, devant la concurrence, à fonder un Prix du Roman, l'a emboîté dans la série dont elle avait la pratique, la série Monthyon, en a fait un prix d'excellence Monthyon. Tenons-nous en aux spécialités; de ce qu'un fabricant a la bonne marque de cassis, n'allons pas lui acheter notre guignolet. (Thibaudet, 1925)

12 De nombreux prix comme le prix Fémina en 1904 en réaction à la misogynie du Goncourt lui ont succédé, mais c'est surtout le prix Renaudot, en 1926, et le prix Interallié en 1930 créés par des journalistes, qui mettent à mal le modèle académique (Ducas, 2010) en proposant des instances de légitimation directement issues de la sphère médiatique affirmant l'entrée de la littérature dans le champ de la grande production. Le prix Médicis créé en 1958 s'insurgeait quant à lui contre tous les autres prix considérés comme conformistes.

13 L'ensemble de ces prix, les "Prix d'automne », obéit à des logiques contradictoires : une logique littéraire et symbolique du coté du pôle de production restreinte de la littérature longtemps à la charge de la reconnaissance de l'État et une logique économique tendant vers le pôle de grande production et de la reconnaissance par les pairs. Le passage du mécénat littéraire aux industries culturelles est révélateur de la mutation de l'écosystème dont la littérature fait partie. La figure classique héritée de l'Ancien Régime s'est progressivement vue remplacée par l'image, ou les images contemporaines d'auteurs vus comme des « professionnels du livre » ou des vedettes de la littérature, tout du moins par les éditeurs et les libraires qui feront de la littérature un événement dès les années 1920 (Ducas, 2010)

14 La diversification de la culture et l'émergence de «niches » de marché à fait naître de nouvelles instances de légitimation littéraire : les « anti-prix » populaires, formés par des jurys d'amateurs ou instaurés par les médias (radios, télévisions, magazines); ils constituent une forme d'expertise littéraire que Claude Poliak désigne comme " profane " (Poliak, 2006), en vue, en théorie tout du moins, d'élargir l'éventail des genres récompensés et d'échapper à la consanguinité jury-éditeur ou éditrice-critique littéraireécrivains.

15 Mais en réalité, les effets des prix sont paradoxaux: si on peut les voir comme les régulateurs d'une hyper-offre du marché, ils semblent également perpétuer une certaine idée de la littérature qui dicte un canon dont la matrice est pour une large part européenne. Dans cette perspective, l'attribution du «certificat d'universalité » (Casanova, 2008: 217) que représente le prix Nobel de littérature un à des auteurs français semble fonctionner comme légitimation internationale de leur consécration dans le champ littéraire français. 


\section{Le Nobel, un prix polémique}

16 Le prix Nobel de Littérature, attribué pour la première fois en 1902, est un des plus anciens prix littéraires internationaux et peut-être le plus prestigieux et le plus polémique. $\mathrm{Vu}$ l'importance économique $\mathrm{du}$ Prix et sa portée mondiale, il est systématiquement objet de discussions et critiques. Si on lit la liste des cent onze lauréats entre 1901 et 2015 on comprendra facilement pourquoi.

Dès que l'on regarde cette liste, on ne peut que souligner l'absence scandaleuse de plusieurs « géants » de la littérature mondiale : Léon Tolstoï, Marcel Proust, James Joyce, Virginia Woolf, Emile Zola, Nabokov et plusieurs autres écrivains, centraux dans l'histoire littéraire, soit n'ont jamais reçu de nomination (c'est le cas de Joyce), soit en ont reçu plusieurs (Tolstoï, par exemple, en a reçu dix-neuf...). Ils ont toujours été écartés en faveur d'écrivains vus aujourd'hui comme « mineurs » dans le canon occidental. Malgré son ambition (et son statut) de "Prix des Prix ", le Nobel de Littérature, a toujours été un lieu politique où se jouent des intérêts de prestige, visibilité et affirmation internationale tendant à renforcer un statu quo esthétique et politique à prédominance européenne (du nord).

\section{Prix Nobel « français » ou « en français »?}

Dans la lutte pour le prestige international que constitue la «course » au Prix Nobel, le classement des lauréats par pays dépend donc de manière déterminante des critères, ainsi que des intérêts, des pays responsables de ce classement ${ }^{1}$. C'est ainsi que d'après la web page officielle du Prix Nobel et la généralité de la presse internationale, la langue française est considérée à l'unanimité comme la deuxième langue des Nobel de Littérature (avec quinze Prix), à la suite de l'anglais (trente Prix)². Mais cette unanimité cesse d'exister quand on énonce les pays d'où proviennent ces œuvres ou leurs auteurs. Ainsi des trente prix décernés à des écrivains anglophones, dix ont été remportés par des écrivains anglais, dix par des auteurs américains, quatre par des irlandais, les autres étant distribués entre plusieurs anciennes colonies britanniques (Afrique du Sud, Canada, Australie, Inde).

Des cent-onze Prix Nobel de Littérature distribués entre 1901 et 2014, la France en aura gagné onze ou quinze selon les sources et les points de vue : quinze pour la majorité des sources françaises et quelques journaux étrangers; onze sur la page web du Prix Nobel (qui liste les auteurs par "pays d'origine») et dans la majorité de la presse écrite en anglais.

On peut ainsi constater des confusions systématiques entre la langue des œuvres et le pays d'origine des auteurs, ces mélanges contribuant à renforcer des inégalités qui dépassent largement la littérature proprement dite. Ces amalgames de critères et ces ambiguïtés, littérature "anglaise » vs. en anglais, ou littérature "française »vs. en français par exemple, mènent d'ailleurs à des résultats pour le moins paradoxaux.

21 C'est ainsi que du point de vue de la presse et des discours officiels francophones, l'inclusion de Gao Xinjian (Prix Nobel 2000), écrivain d'origine chinoise naturalisé français, dans la liste d'auteurs "français » est tout à fait légitime, bien qu'il écrive en chinois des romans consacrés à la République Populaire de Chine. Dans le site officiel du 
Prix Nobel, inversement, cet écrivain est considéré comme chinois puisqu'il est né en Chine. Suivant ce même principe, des écrivains comme Albert Camus, St. John Perse et Claude Simon, nés en Algérie, Guadeloupe et Madagascar respectivement, ne sont pas non plus considérés comme français. Par ailleurs, Samuel Beckett (Prix 1969) qui, comme on s'en souvient, a vécu en France pendant de longues années et qui a écrit en français une partie très importante de son œuvre apparaît dans un des onglets de la page web comme " auteur de langue française » et dans un autre comme " écrivain irlandais » (ibid.). Pour ceux que Casanova désigne comme « écrivains internationaux »(Casanova, $2008: 224)$, les «nationalités » oscillent, suivant le capital symbolique des différents pays et selon des critères politiques, entre autres.

En ce qui concerne donc les Nobel français de Littérature, entendus comme de nationalité française au moment du prix, on peut observer que parmi l'ensemble des quinze lauréats, dix ont été primés avant 1960 et les cinq autres dans les cinquante années suivantes. Ces deux phases correspondent à des moments différents de l'influence française sur le plan international, en littérature comme dans bien d'autres domaines. A la suite de l'attribution du Prix à Jean-Paul Sartre en 1964, que l'écrivain a refusé, comme on le sait, la France allait devoir attendre plus de vingt ans pour recevoir à nouveau le Prix Nobel de Littérature, concédé à Claude Simon en 1985, Gao Xinjian suivra en 2000, Le Clézio en 2008 et enfin Patrick Modiano en 2014.

Les messages de l'Académie Suédoise justifiant chaque année l'attribution du Nobel Littérature se sont eux aussi transformés au fil du temps. Bien que conservant la rhétorique et l'affirmation des critères fondamentaux du Prix, ces messages ont évolué d'année en année, de la valorisation générale de la «condition humaine » dans la première moitié $\mathrm{du} \mathrm{xx}^{\mathrm{e}}$ siècle (dédoublée selon les années et les lauréats en « drames de la vie humaine ", "conflits humains", " conscience humaine", "sympathie humaine", etc.) à une rhétorique plus concrète et située, historiquement et géographiquement, à partir des années 60 . On a alors apprécié la « conscience approfondie du temps dans le portrait de la condition humaine" chez Claude Simon; la "recherche de la vérité et influence dans l'époque » chez Sartre ; vu en Le Clézio l'« explorateur d'une humanité audelà et en-dessous de la civilisation régnante » et trouvé chez Modiano «l'art de la mémoire avec laquelle il a évoqué les destinées humaines les plus insaisissables » et "dévoilé le monde de l'occupation ». Les changements subtils dans les messages de présentation des lauréats, plus concernés par le monde contemporain au cours des dernières décennies, semblent correspondre eux aussi aux changements du système littéraire dont le Prix Nobel fait partie.

Après la lente disparition du milieu littéraire de la Belle Epoque, la littérature en France a connu une période exceptionnelle dans les années 50, notamment du point de vue politique, avec l'écrivain engagé, représenté par Jean-Paul Sartre. Cette position sera refusée par Roland Barthes ou Maurice Blanchot, par exemple, ainsi que par des écrivains qui considéraient la négativité, l'expérimentalisme et la recherche de neutralité comme l'essence de la littérature, et prônaient un engagement à travers l'écriture littéraire, c'està-dire à travers la forme. C'est le cas des écrivains du Nouveau Roman dont fait partie Claude Simon. Le problème de l'engagement croise ainsi des questions poétiques et esthétiques, confrontant la vision de la littérature en tant que lecture et dialogue dans l'espace de la polis à une expérience d'écriture moderne, solitaire et tragique, témoignage de la condition de l'écrivain partagé entre condition sociale et vocation intérieure. Cette approche prolongeait ainsi l'histoire du conflit entre littérature et monde, ainsi que la 
notion d'autonomie de la littérature, que Gustave Flaubert avait commencée au XIX siècle (Compagnon, 2007 : 556).

La conscience textuelle et la critique du langage dans ses rapports au monde que cherchait le Nouveau Roman auront une importance décisive pour la génération suivante dont les romans, construits de manière plus « classique » et beaucoup plus lisibles, posent maintenant des questions explicitement liées à la conscience historique ainsi qu'à la mondialisation contemporaine. Du multiculturalisme de Le Clézio aux récits et aux mémoires problématiques de Modiano, si les préoccupations de ces écrivains passent par l'interrogation du monde et de la mémoire sans remettre vraiment en question le langage qui les construit, elles passent aussi très visiblement par la forme de leurs récits, par les choix thématiques, par les modes de construction des romans qui les supportent.

L'attribution du Prix Nobel à des auteurs "français» dans les dernières décennies, notamment à partir des années 60 , constitue un exemple probant de l'évolution du système littéraire pendant cette période, notamment en ce qui concerne la revendication d'une position individuelle engagée et critique de l'écrivain (c'est le cas de Sartre et de Claude Simon) et la transition vers une professionnalisation du métier d'écrivain, intégré dans l'industrie des media et dans le marché éditorial global, comme c'est le cas de JeanMarie Gustave Le Clézio et de Patrick Modiano.

\section{Le prix Nobel et les prix littéraires français}

La vocation internationale du prix Nobel fait de celui-ci un phénomène assez différent des autres prix littéraires notamment français : d'une part, cette vocation internationale se superpose largement aux rivalités éditoriales nationales et, d'autre part, les critères définis par le Nobel sont assez spécifiques, privilégiant globalement l'idéalisme de ce que l'on désigne comme « œuvre », non nécessairement d'un livre en particulier et, surtout, la position éthique dont elle découlerait. Dans la pratique, le dilemme du Nobel semble bien celui de l'oscillation entre la reconnaissance d'une œuvre consacrée, au moins en occident (écrite en anglais, français ou espagnol de préférence) et le désir de faire connaître des œuvres considérées comme de qualité par le jury, même si elles restent plus ou moins confidentielles auprès du public. Les questions liées à l'accessibilité des œuvres, comme la capacité à en assurer la diffusion et la traduction en plusieurs langues, semblent assez clairement jouer un rôle central dans les critères du jury du Nobel (Casanova, $2008: 231$ ).

En tous les cas, pour ce qui est des écrivains français, à l'exception de Gao Xinjian, choix nettement politique dû en grande partie à la plus grande visibilité de ses œuvres après son exil en France, le Prix Nobel apparaît presque toujours comme la consécration d'une œuvre qui est déjà largement reconnue et internationalisée, soit parce qu'elle représente un courant artistique et philosophique devenu international, c'est le cas de Jean-Paul Sartre ou de Claude Simon, soit parce qu'elle engage une carrière littéraire pleinement légitimée par le système littéraire, notamment par l'accumulation de prix littéraires, comme pour Le Clézio et Modiano.

Pour un grand nombre de ces écrivains, le Prix Nobel succède à différents prix français : avant le Nobel, Claude Simon avait déjà obtenu le prix Médicis en 1967. Curieusement, le Nobel de Claude Simon lui fut attribué une année après l'attribution du Goncourt à Marguerite Duras, contribuant ainsi assez efficacement à la consécration internationale 
du Nouveau Roman dans le système littéraire. Patrick Modiano, de son côté, avait accumulé avant 2014 onze prix, français et européens dont le Goncourt et le Grand Prix du Roman de l'Académie Française, le prix Jean Monnet de littérature européenne et le Prix Autrichien de Littérature. En 2008 Jean-Marie Gustave Le Clézio avait remporté neuf prix dont quatre internationaux (Union Latine et Stig Dagermann par exemple) ainsi que le prix du public France Télévisions. Cette sorte d'articulation entre prix antérieurs et prix Nobel, surtout ces dernières années, semble donc témoigner d'une interdépendance globale croissante du système littéraire.

Quant aux critères du jury de Stokholm (ou tout au moins d'une partie importante de ce jury) concernant le choix d'auteurs français, ils semblent être fondés sur un principe que l'on pourrait taxer de "représentativité paradigmatique»: le paradigme de l'engagement, d'une part, développé par les auteurs appartenant à la génération de la deuxième guerre mondiale. Sartre, tout d'abord, représentant de l'homme intégré dans un monde socialement conflictuel et confus, Claude Simon, ensuite, penché sur les difficultés de compréhension et d'expression d'une réalité complexe et difficile à lire, construite au départ par le langage, avec toutes ses ambiguïtés et ses limitations. Les choix $\mathrm{du} \mathrm{xxI}^{\mathrm{e}}$ siècle retombant sur Jean-Marie Gustave Le Clézio et Patrick Modiano s'intègrent apparemment dans une approche post-moderne, en réaction contre la génération précédente. Le Clézio, auteur de romans au langage clair et lisible où le regard est tourné vers l'ailleurs, vers d'autres réalités et d'autres paysages ouvrant le roman français à la «littérature-monde» semble représenter une sorte de retour au « mainstream » contrastant avec la complexité d'écriture et de lecture de l'approche d'un Claude Simon par exemple. Patrick Modiano et son approche psychologique des « oubliés » de l'Histoire, dans des romans sans héros qui, à rebours des récits officiels sur la Résistance, passent de manière assez ambigüe de la voix des héros (ou anti-héros) à celle des collabos donne une voix à des personnages invisibles et sans qualité, « honteux ", absents des discours officiels. Pour Per Wästberg, président du comité Nobel, Modiano serait « le meilleur écrivain français vivant », un avis partagé par le secrétaire permanent du comité, Peter Englung, qui qualifie Modiano de «Marcel Proust moderne». Ces avis laudatifs ont cependant été relativisés par la presse internationale, appelant parfois l'écrivain "Patrick Who ${ }^{3}$ pour souligner une certaine perplexité quant au choix d'un lauréat finalement assez peu connu sur la scène littéraire internationale...

31 La "vraie reconnaissance internationale" des écrivains français, la "capacité à renouveler la fiction narrative en langue française » ou «la prégnance de la langue française »dans l'actuelle mondialisation que les deux derniers Nobel de littérature démontreraient sont donc à considérer en tenant compte du fait que «l'activité des instances consacrantes est une opération ambiguë (...) [qui] s'exerce aussi, de façon presque inévitable, selon les normes de celui qui 'juge'»(Casanova, 2008: 226). La dynamique des prix littéraires français sera sans doute sinon modifiée tout du moins défiée par l'arrivée du québécois d'origine haïtienne Dany Laferrière à l'Académie Française ou du franco-congolais Alain Mabankou au collège de France, représentants de ce qui serait une nouvelle dimension de l'universalité de la langue française, la dimension transnationale et décentrée de cette langue au passé prestigieux et des littératures qui s'expriment en son sein. 


\section{BIBLIOGRAPHIE}

Blanckeman, Bruno. (2010-2011). « D'un Nobel l'autre » [on-line], Carnets 2010-2011, pp. 257-265.

Portugal : [disponible le 1/11/2015]

< URL : http://revistas.ua.pt/index.php/Carnets/article/view/827/759>.

COMPAGNON, Antoine (2007). « Xx $x^{\mathrm{e}}$ siècle », in Michel Delon, Françoise Mélonio, Bertrand Marchal, Jacques Noiray, Antoine Compagnon (orgs). La littérature française : dynamique et histoire II. Paris : Gallimard, col. Folio Essais Inédit.

DuCAS, Sylvie (2006). «Prix Goncourt et reconnaissance littéraire, stratégies d'accès à la consécration ", in Gérard Mauger, (org.). Droits d'entrée, Modalités et conditions d'accès aux univers artistiques. Paris : Éditions de la Maison des Sciences de l'homme.

DucAs, Sylvie (2010). « Prix littéraires en France : consécration ou désacralisation de l'auteur?» [on-line], COnTEXTES. France : [disponible le 22/06/2015]

< URL : http://contextes.revues.org/4656 ; DOI : 10.4000/contextes.4656>.

DuCAS, Sylvie (2013). La Littérature à quel(s) prix ? Histoire des prix littéraires. Paris : La Découverte.

CASANOVA, Pascale (2008). La république mondiale des lettres. Paris : Seuil.

PoliAK, Claude. (2006). Aux frontières du champ littéraire. Sociologie des écrivains amateurs. Paris : Économica.

« Pour une 'littérature monde' en français » [on-line], Le Monde des livres, 15.03.2007. [disponible le $29 / 09 / 2015]$

< URL : http://www.lemonde.fr/livres/article/2007/03/15/des-ecrivains-plaident-pour-unroman-en-francais-ouvert-sur-le-monde_883572_3260.html>.

THIBAUDET, Albert (1925). « Du Journal des Goncourt », Nouvelle Revue française, tome xxv, décembre 1925, pp. 741-742.

SILVA, Marie-Manuelle (2012). « Banda desenhada ou literatura desenhada ? », in Eunice Ribeiro, (orgs), Modenidades comparadas. Estudos literários/Culturais Revisitados. Famalicão : Humus EdicõesCEHUM, pp. 79-88.

STIÉNON, Valérie (2010). « La consécration à l'envers. Quelques scénarios physiologiques (1840-1842)» [on-line], in COnTEXTES 7/2010, France : [disponible le 1/11/2015] < URL : http://contextes.revues.org/4654 ; DOI : 10.4000/contextes.46542010>.

VIALA, Alain (1985). Naissance de l'écrivain. Sociologie de la littérature à l'âge classique. Paris : Minuit. VIART, Dominique (2014). Entretien [on-line], in Le Temps 10/10/2014, France : [disponible le 22/06/2015].

< URL: http://www.letemps.ch/culture/2014/10/09/une-reconnaissance-va-plus-oeuvre-quecrivain. Consulté>. 


\section{NOTES}

1. Comme le rappelait Pascale Casanova en 1999, les critères de l'académie du Nobel ont d'abord été politiques (de la prudence diplomatique neutre à l'engagement actif) puis du côté de la réception, visant une certaine universalité et par là même le public le plus large possible (Casanova, $2008: 200-201$ ).

2. Un exemple parlant de cette situation serait l'ensemble des lauréats pendant les années de nomination de Tolstoï, relativement peu connus aujourd'hui : Theodor Mommsen, historien allemand (1902) ; Bjørnstjerne Bjørnson, poète norvégien (1903) ; Frédéric Mistral, poète français et José Echegaray, dramaturge espagnol (1904) ; Henrik Sienkiewicz, écrivain polonais (1905).

3. Robson, Leo « Why nobody knows what to think about Patrick Modiano winning the Nobel Prize for Literature" in: Newstatesman, 8/10/2014 http://www.newstatesman.com/ culture/2014/10/why-nobody-knows-what-think-about-patrick-modiano-winning-nobel-prizeliterature

\section{RÉSUMÉS}

Dans la « nation littéraire » Française, les prix prolifèrent dans ce que Sylvie Ducas (2006) désigne comme une économie du prestige fondée, d'une part, sur des mythologies associées aux écrivains et, de l'autre, sur des formes et des modalités de définition de la "valeur littéraire ». Cet article se propose d'examiner quelques-unes des interactions entre le phénomène littéraire que représente le prix Nobel et le champ littéraire français et de problématiser la transformation de la figure classique de l'écrivain héritée de l'Ancien Régime, dont la fonction sociale a perdu de son prestige, à la lumière des rhétoriques et de modèles auxquelles s'adossent l'attribution du prix Nobel de littérature depuis Sartre en 1964.

In the French « literary nation ", prices proliferate in what Sylvie Ducas (2006) designates as an economy of prestige based, on the one hand, on mythologies associated with writers and, on the other, on ways of defining «literary value ». This paper examines some of the interactions between the literary phenomenon represented by the Nobel Prize and the French literary field, and analyzes the transformation of the classical idea of the writer inherited from the Ancien Régime through the rhetoric and models supported by the Nobel Prize for Literature since the award of the Prize to Sartre in 1964.

\section{INDEX}

Mots-clés : Prix Nobel de littérature, champ littéraire, littérature en Français, littérature internationale

Keywords : Nobel Prize in Literature, literary field, literature in French, international literature 


\section{AUTEURS}

\section{MARIE-MANUELLE DA SILVA}

Université du Minho

mmcsilva[at]ilch.uminho.pt

\section{EDUARDA KEATING}

Université du Minho

mekeating[at]ilch.uminho.pt 